\title{
Publisher Correction: Self-renewing resident cardiac macrophages limit adverse remodeling following myocardial infarction
}

Sarah A. Dick, Jillian A. Macklin (D), Sara Nejat D, Abdul Momen, Xavier Clemente-Casares, Marwan G. Althagafi, Jinmiao Chen D, Crystal Kantores Di , Siyavash Hosseinzadeh, Laura Aronoff, Anthony Wong (D), Rysa Zaman (D), Iulia Barbu, Rickvinder Besla, Kory J. Lavine, Babak Razani, Florent Ginhoux (D), Mansoor Husain, Myron I. Cybulsky,

Clinton S. Robbins and Slava Epelman (D)

Correction to: Nature Immunology https://doi.org/10.1038/s41590-018-0272-2 (2018), published online 11 December 2018.

In the version of this article initially published, the equal contribution of the third author was omitted. The footnote links for that author should be "Sara Nejat ${ }^{1,11}$ " and the correct statement is as follows: "11 These authors contributed equally: Sarah A. Dick, Jillian A. Macklin, Sara Nejat." The error has been corrected in the HTML and PDF versions of the article.

Published online: 12 March 2019

https://doi.org/10.1038/s41590-019-0363-8

\section{Publisher Correction: SERPINB1-mediated checkpoint of inflammatory caspase activation}

Youn Jung Choi, Stephanie Kim, Younho Choi, Travis B. Nielsen, Jun Yan, Alvin Lu, Jianbin Ruan, Hye-Ra Lee, Hao Wu, Brad Spellberg and Jae U. Jung (D)

Correction to: Nature Immunology https://doi.org/10.1038/s41590-018-0303-z (2019), published online 28 January 2019.

In the version of this article initially published, the label (CASP4- $\mathrm{C}_{285} \mathrm{~A}-\mathrm{HA}$ ) above the second and fifth lanes in the right blot in Fig. 1e is incorrect; the correct label is CASP4- $\mathrm{C}_{258} \mathrm{~A}-\mathrm{HA}$. Also, the two labels at right above the plot in Fig. $6 \mathrm{c}$ were switched; the far right label should be 'Co-housed Serpinb1 $a^{-- \text {- }}$ (in red font) and the label just to its left (above the fourth column) should be 'Co-housed WT' (in black font). Finally, the bottom two symbols in the key to Fig. 7d were switched; the red circle should identify 1CARD-SUMO (TEV) and the blue triangle should identify 1CARD-SUMO + SERPINB1 (TEV). The errors have been corrected in the HTML and PDF versions of the article. 